\title{
ESTUDO DE COMPÓSITOS POLIMÉRICOS BASEADOS EM POLIPROPILENO RECICLADO VISANDO APLICAÇÃO EM PLACAS PARA COLETORES DE ENERGIA SOLAR
}

\author{
Hilton de Souza Rutsatz ${ }^{1}$ \\ Cláudia Mendes Mählmann ${ }^{2}$ \\ Adriane Lawisch Rodríguez.
}

\begin{abstract}
RESUMO
Considerando que a demanda de energia limpa vem crescendo no decorrer dos anos, este estudo objetivou a utilização de plásticos reciclados para a composição de partes de coletor solar, substituindo materiais tradicionais como alumínio e cobre. Para tanto foram produzidos compósitos poliméricos com propriedades interessantes e adequadas para este fim. Neste artigo, são apresentados os resultados de absorção de calor por placas de compósitos poliméricos baseados em polipropileno (PP) reciclado como matriz e como fase dispersa negro de fumo, pó de alumínio e pó de fumo. Além disto, avaliou-se a utilização de compatibilizante, anidrido maléico $(2 \%, \mathrm{~m} / \mathrm{m})$. Foram realizados ensaios de determinação de absorção de calor, considerando o gradiente de temperatura através das placas de compósitos poliméricos, tendo as formulações com pó de fumo apresentado os melhores resultados, principalmente em conjunto com pó de alumínio.
\end{abstract}

Palavras-chave: Polipropileno reciclado. Compósitos. Coletor de energia solar.

\section{ABSTRACT}

Considering that the demand for clean energy has been growing over the years, this study aimed to use recycled plastics for the composition of solar collector parts, replacing traditional materials such as aluminum and copper. Thus, polymer composites potentially interesting properties for this purpose were produced. In this paper, the results of heat absorption by leaves made of polymeric composites based on recycled polypropylene (PP) as matrix and dispersed phase as carbon black, aluminum powder, and tobacco powder are presented. Moreover, the use of a compatibilizer, maleicanhydride $(2 \%, \mathrm{w} / \mathrm{w})$, was evaluated. Tests to determine heat absorption were performed, considering the temperature gradient across the plates of polymer composites. The composites with tobacco powder attained the best results, mainly with the aluminum powder.

Keywords: Recycled polypropylene. Composites. Solar energy collector.

\footnotetext{
1 Acadêmico do Curso de Engenharia Mecânica da Universidade de Santa Cruz do Sul - UNISC. $<$ hiltonrutsatz@gmail.com>

${ }^{2}$ Mestre em Ciências - área de concentração em Física, docente do Departamento de Química e Física da Universidade de Santa Cruz do Sul - UNISC. <mclaudia@unisc.br>

3 Doutora em Engenharia, docente do Departamento de Engenharia, Arquitetura e Ciências Agrárias da Universidade de Santa Cruz do Sul - UNISC. <adriane@unisc.br>
} 


\section{INTRODUÇÃO}

A energia solar é a fonte renovável menos poluente conhecida até o momento. Esta forma ainda não é aproveitada eficientemente ao máximo possível. Providenciar um sistema com grande eficiência é uma tarefa que encontra algumas dificuldades e certo grau de complexidade. Atualmente, sistemas geradores de energia são mais utilizados no aquecimento residencial, em sistemas térmicos e fotovoltaicos (COMETTA, 1978).

O sistema térmico consiste na conversão de radiação solar em calor, usando a energia conforme a aplicação desejada, foco do estudo. O sistema fotovoltaico converte a energia radiante do sol em energia comum. A energia térmica estudada é de baixa temperatura, na escala máxima de até $100^{\circ} \mathrm{C}$, tendo emprego como exemplo no aquecimento de ambientes, aquecimento de água residencial e piscinas, sendo uma aplicação mais simples e imediata.

As aplicações da energia solar em baixa temperatura, para aquecimento de água em piscinas, processos de secagem e certos processos industriais, podem ser obtidas através de coletores planos abertos ou fechados ou por coletores de tubos evacuados, podendo ser operados por água como seu fluído de trabalho. Sua aplicabilidade é bastante adequada por ocorrer a maior oferta de energia em dias ensolarados de verão. Um sistema de aquecimento solar se divide em três subsistemas: captação, armazenamento e consumo.

A presente pesquisa segue duas linhas de estudo, uma para o desenvolvimento do boiler (reservatório do fluido) e outra para as placas coletoras. Neste trabalho são apresentados os resultados alcançados da formulação e processamentos das placas coletoras alternativas, as quais foram elaboradas em compósitos poliméricos envolvendo materiais reciclados com adição de fase dispersa. Também é apresentada a avaliação na captação de calor a partir da sua utilização. Os ensaios realizados foram de bancada, possibilitando a aquisição de dados de temperatura em intervalos de tempo.

\section{FUNDAMENTAÇÃO TEÓRICA}

O sol, fonte de energia renovável, serve tanto para captação de calor como fonte de luz, tornando-se a alternativa energética mais promissora para o futuro. A energia solar utilizada para aquecimento de água ou geração de energia é a solução para regiões mais afastadas ou áreas autossuficientes.

Dados obtidos, apresentados na Tabela 1, demonstram a comparação entre as fontes energéticas. 
Tabela 1 - Comparação de fontes energéticas para cada $\mathbf{m}^{2}$ de coletor solar instalado.

- Economia de $55 \mathrm{~kg}$ de gás liquefeito de petróleo (GLP) anual;

- Economia de 66 litros de diesel anual;

- Proteção de cerca de 56 metros quadrados $\left(\mathrm{m}^{2}\right)$ de terras alagadas para a geração de energia elétrica;

- Economia $215 \mathrm{~kg}$ de lenha anual.

Fonte: ASTROSOL 2012

O Brasil possui um dos maiores potenciais para utilização da energia solar e a utilização é pouca perto do potencial oferecido. (SOLBRASIL, 2013). Os sistemas de aquecimento solar mais utilizados dividem-se em duas linhas: circulação forçada e termossifão. A diferença de trabalho é notada em densidade do fluido e auxílio de bombas eletrocirculadoras para movimento do fluido. O sistema por termossifão tem grande uso, funcionando na variação da densidade do fluido através da convecção de calor obtida na placa, quanto mais aquecido o fluido, menor será sua densidade. Isto provoca uma circulação natural no interior do sistema, este por si consistido em coletor solar, depósito acumulador (boiler), purgador, vasos de expansão e outros pequenos acessórios (PORTAL DAS ENERGIAS RENOVÁVEIS, 2004).

O coletor solar tem como funcionalidade aquecer o fluido através da circulação no interior da placa. Este aquecimento é proveniente da radiação solar ao ultrapassar as camadas de vidro colidi nas aletas com pigmentação escura. Forma-se um efeito estufa no interior do coletor, sendo aquecido por convecção o fluido que circula dentro de tubulações de cobre (SOLETROL, 2013).

Na Figura 1 é apresentado o esquema de composição e demonstração de funcionamento do sistema de captação de energia e armazenamento de água aquecida. Este sistema é mais utilizado para temperaturas menores que $100^{\circ} \mathrm{C}$, empregados em aquecimento de piscinas e chuveiros domiciliares. Estudos realizados indicam a possibilidade de fabricação em massa de coletores solares de baixo custo utilizando materiais alternativos na composição do sistema (RUSSEL et al., 1982). Além disso, filmes poliméricos foram propostos para a composição de coletores de baixo custo (TSILINGIRIS et al., 1997). 
Figura 1 - Coletor solar de placa horizontal.

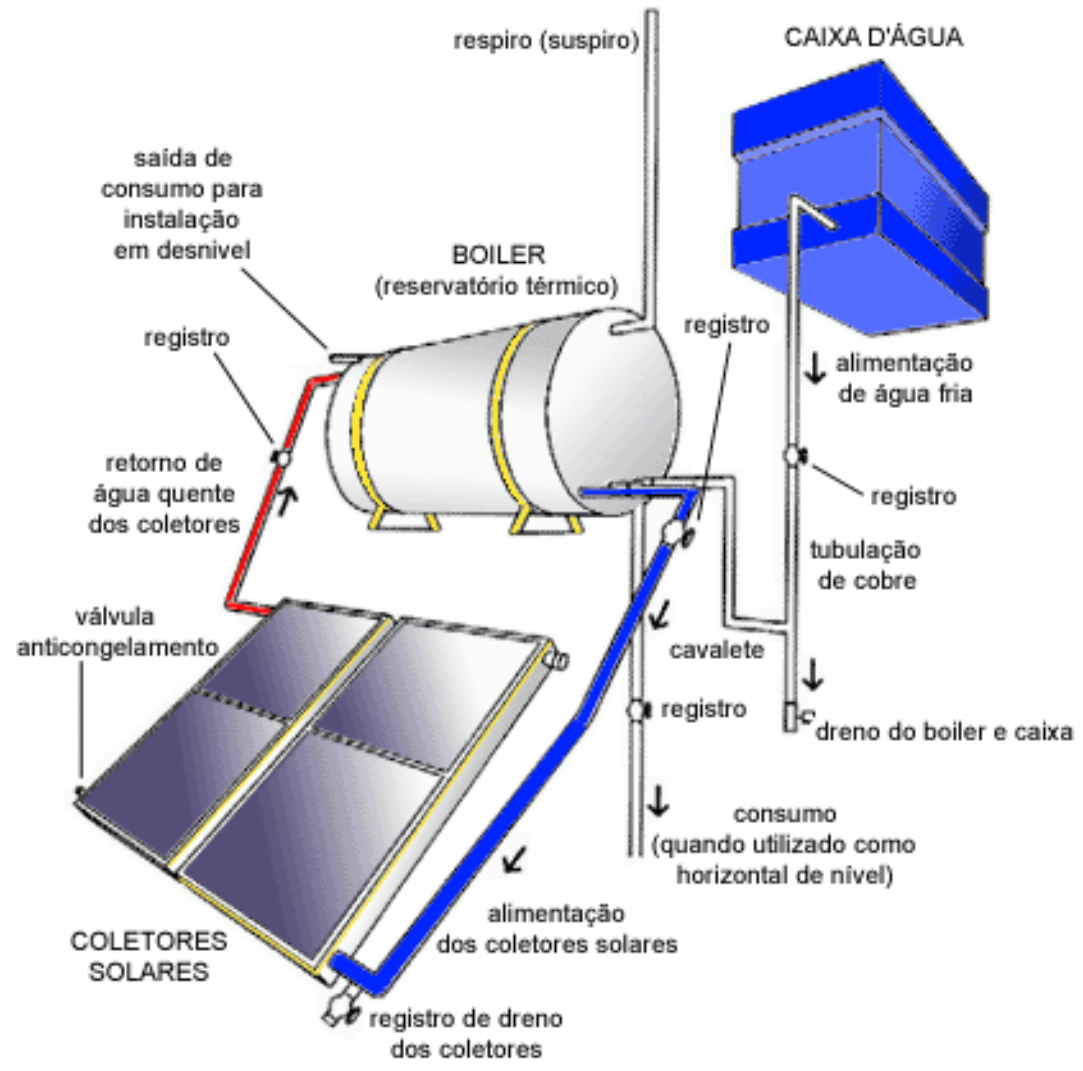

Fonte: SOLETROL, 2013

\section{METODOLOGIA}

Foram processadas placas coletoras elaboradas a partir de compósitos poliméricos baseados em polipropileno (PP) reciclado. O PP pós-consumo utilizado foi o proveniente de embalagens de água mineral, onde foi realizada a moagem do polímero, seguida da realização da mistura com os componentes da fase dispersa através da homogeneização dos componentes. Na Figura 2 é apresentado o fluxograma do processo de preparação do PP para utilização como matriz dos compósitos estudados.

\section{Figura 2 - Embalagem de PP, moinhos de facas, placa polimérica de PP puro.}

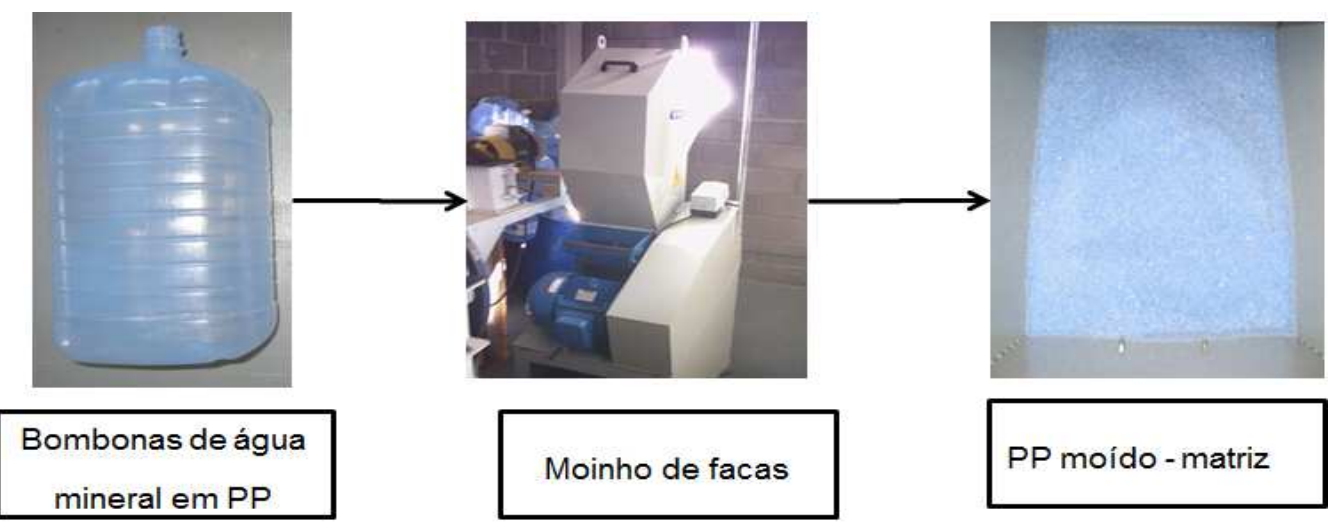


Como componentes da fase dispersa foram utilizados o negro de fumo (furnex N762), o pó de alumínio (StandartChromal VIII - Clariant) e/ou o pó de fumo. Além disto, foi avaliada a utilização de compatibilizante, anidrido maleico, em uma porcentagem de $2 \%$ $(\mathrm{m} / \mathrm{m})$, utilizando-se o processo de homogeneização, propiciando assim a graftização deste à cadeia polimérica. Esta adição foi realizada com o objetivo de melhorar a adesão interfacial entre a matriz polimérica e os componentes da fase dispersa (MOAD et al., 1999; PASSAGLIA et al., 2014).

Para a obtenção das placas absorvedoras de calor, os componentes da fase dispersa foram utilizados separadamente nas quantidades de $6 \%(\mathrm{~m} / \mathrm{m})$ de negro de fumo, $20 \%(\mathrm{~m} / \mathrm{m})$ de pó de fumo e $10 \%(\mathrm{~m} / \mathrm{m})$ de uma mistura de negro de fumo com pó de alumínio.

O estudo realizado objetivou a criação de uma placa com PP reciclado com dimensões de 400x400 mm com espessura de 4 mm, sendo de fácil manutenção, utilizando uma composição visando à boa eficiência na captação de calor, bem como baixo custo e baixa dispersão de calor. A construção se realizou com a moagem das embalagens em moinho de facas (Mecanofar - MF300) e após foram selecionados os componentes e adicionados em porcentagem ao peso da placa. Estas misturas de PP moído com negro de fumo, pó de fumo e pó de alumínio, foram preparadas no homogeneizador MH-100 (MH Equipamentos) para melhor aderência entre os compósitos e polímero moído.

O polímero já misturado foi triturado no moinho, voltando à forma de grânulos, mas já prontos para a compactação em prensa (MH Equipamentos) a $180^{\circ} \mathrm{C}$. Foram realizados ensaios de determinação do fluxo de calor nas placas absorvedoras, nas quais foram medidas temperaturas em diferentes alturas, tendo em vista a possibilidade de aplicação destas como dispositivos coletores em coletor de energia solar.

Na Figura 3 é apresentado o esquema do sistema montado, no qual realizou-se o experimento sobre fluxo de calor nas placas. Através de cinco sensores obtiveram-se as temperaturas nos cinco pontos indicados. Os sensores foram colocados nas distâncias que seguem, a partir da fonte de calor (lâmpada incandescente de 100W): Sensor (spindle) 1 116,6 mm (distância horizontal) e 0 mm (distância vertical); Sensor (spindle) 2 - 138,3 mm (distância vertical); Sensor (spindle) 3- 209,3 mm (distância vertical); Sensor (spindle) 4249,6 mm (distância vertical); Sensor (spindle) 5- 365,8 mm (distância vertical). Ressalta-se que a placa fica entre os sensores 3 e 4 a cerca de $20 \mathrm{~mm}$ em relação a cada um destes sensores. 
Figura 3 - Esquema do sistema utilizado no experimento sobre fluxo de calor.

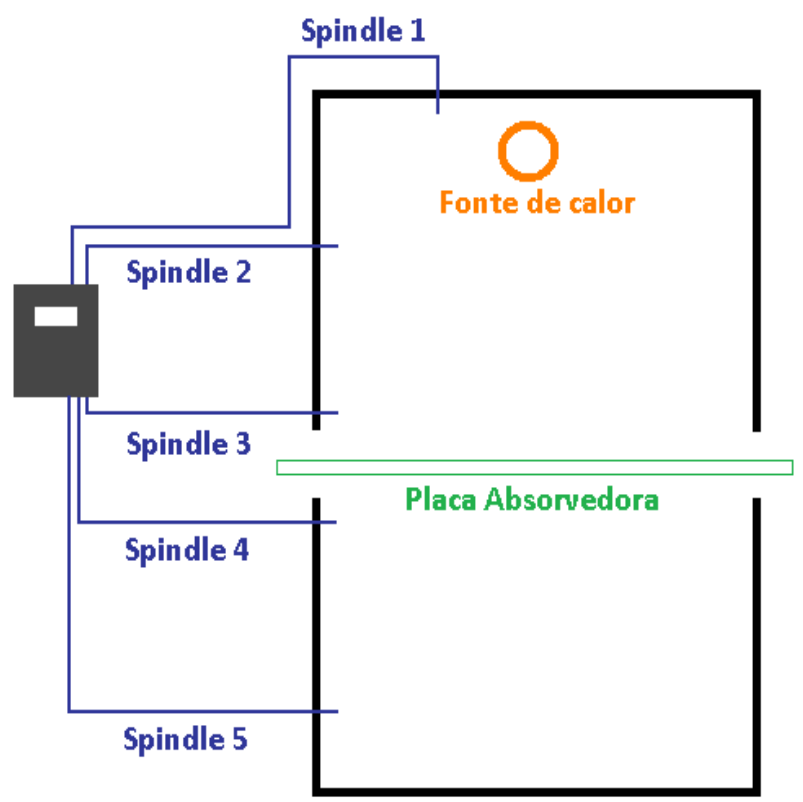

Os sensores de maior importância neste estudo foram os localizados sobre $(209,3$ $\mathrm{mm}$ ) e abaixo da placa $(249,6 \mathrm{~mm})$, que forneceram as informações a respeito da energia absorvida pela mesma. Também foram realizadas as medidas sem a placa no sistema. Estas medidas foram verificadas após $1 \mathrm{~h}$ e $2 \mathrm{~h}$ de experimento, e em triplicata.

\section{RESULTADOS E DISCUSSÕES}

Nas imagens constantes da Figura 4 é apresentado o aspecto visual das placas de compósitos poliméricos (diferentes formulações) obtidas. Na imagem (a) é apresentada a placa de polipropileno puro (PP); na (b), a placa de PP com adição de pó de tabaco; na (c), a placa de PP com adição de negro de fumo; e na (d), da placa de PP com adição de negro de fumo + pó de alumínio.

Já na Tabela 2 são apresentados os resultados das temperaturas medidas nos 5 sensores para os quatro tipos de placas avaliadas. Na Tabela 3 são apresentadas as médias das temperaturas constantes da Tabela 2, bem como o desvio padrão verificado e as temperaturas obtidas na medida realizada sem placa. 
Figura 4 - Aspecto visual das placas absorvedoras estudadas, com diferentes formulações: (a) de PP puro; (b) de PP com adição de pó de tabaco; (c) de PP com adição de negro de fumo; e (d) de PP com adição de negro de fumo + pó de alumínio.
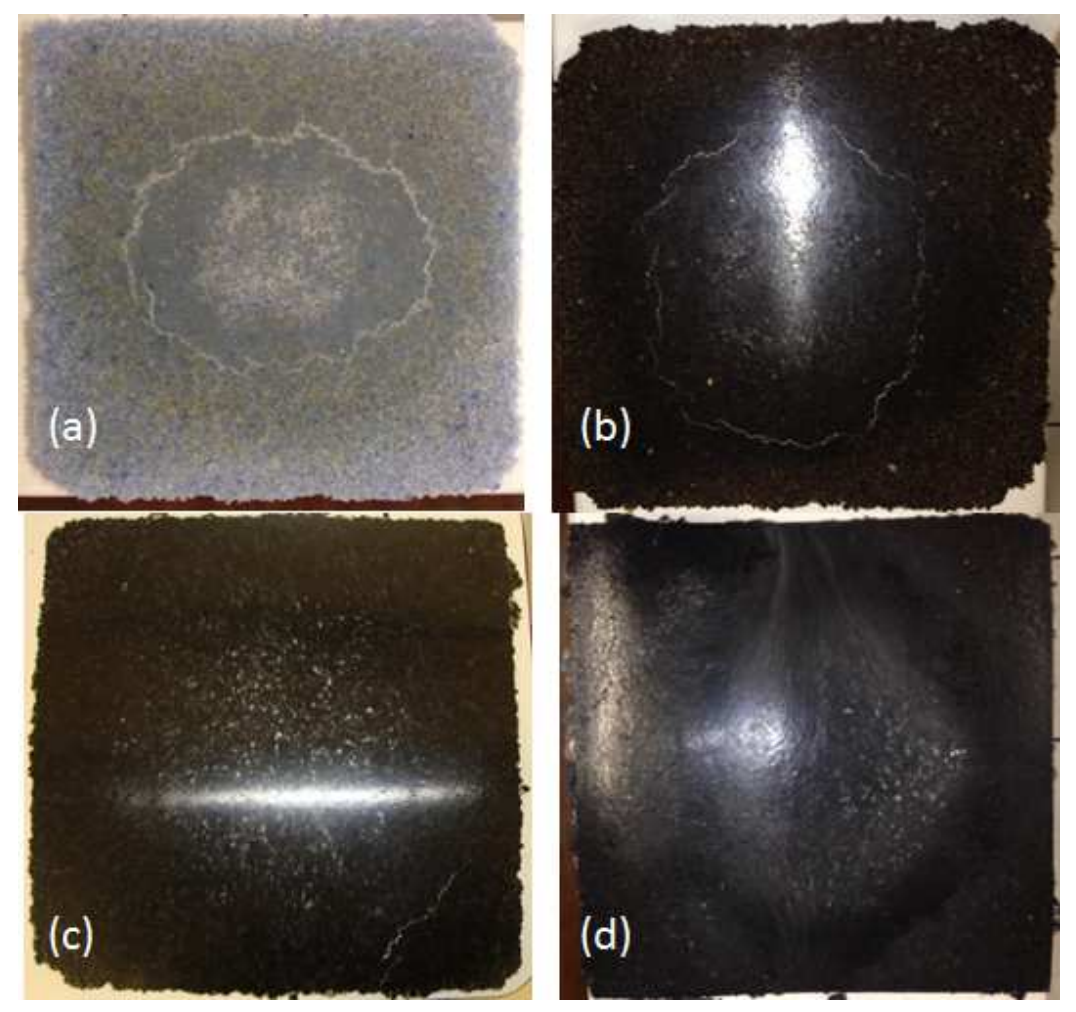

Tabela 2 - Resultados obtidos nas 3 medidas realizadas, nos 5 sensores do sistema experimental para as 4 placas estudadas.

\begin{tabular}{cccccccc}
\hline & & \multicolumn{3}{c}{ PP puro $(\mathbf{1 h})$} & PP + pó de fumo $(\mathbf{1 h})$ \\
\hline & Medidas & $\mathbf{1}\left({ }^{\circ} \mathbf{C}\right)$ & $\mathbf{2}\left({ }^{\circ} \mathbf{C}\right)$ & $\mathbf{3}\left({ }^{\circ} \mathbf{C}\right)$ & $\mathbf{1}\left({ }^{\circ} \mathbf{C}\right)$ & $\mathbf{2}\left({ }^{\circ} \mathbf{C}\right)$ & $\mathbf{3}\left({ }^{\circ} \mathbf{C}\right)$ \\
\hline Sensor 1 & 68,7 & 69,5 & 69,2 & 66,2 & 68,4 & 69,6 \\
Sensor 2 & 57,4 & 58,4 & 57,8 & 54,1 & 56,9 & 58,2 \\
Sensor 3 & 50,2 & 53,6 & 51,3 & 51,1 & 53,9 & 54,8 \\
Sensor 4 & 35,0 & 37,0 & 35,4 & 32,1 & 35,6 & 35,8 \\
Sensor 5 & 28,4 & 29,7 & 28,9 & 24,1 & 27,1 & 27,2 \\
\hline & & \multicolumn{7}{c}{$\mathbf{P P}$ puro $(\mathbf{2 h})$} & \multicolumn{1}{c}{$\mathbf{P P}+$ pó de fumo $(\mathbf{2 h})$} \\
\hline Sensor 1 & Medidas & $\mathbf{1}\left({ }^{\circ} \mathbf{C}\right)$ & $\mathbf{2}\left({ }^{\circ} \mathbf{C}\right)$ & $\mathbf{3}\left({ }^{\circ} \mathbf{C}\right)$ & $\mathbf{1}\left({ }^{\circ} \mathbf{C}\right)$ & $\mathbf{2}\left({ }^{\circ} \mathbf{C}\right)$ & $\mathbf{3}\left({ }^{\circ} \mathbf{C}\right)$ \\
\hline Sensor 2 & 69,3 & 69,8 & 69,6 & 68,1 & 70,0 & 70,1 \\
Sensor 3 & 58,1 & 58,7 & 58,4 & 56,9 & 58,6 & 59,1 \\
Sensor 4 & 53,3 & 53,9 & 53,6 & 54,3 & 55,8 & 55,8 \\
Sensor 5 & 36,6 & 37,3 & 36,9 & 36,3 & 36,8 & 36,9 \\
& 29,3 & 29,7 & 29,7 & 28,7 & 27,7 & 27,7 \\
\hline
\end{tabular}




\begin{tabular}{|c|c|c|c|c|c|c|c|}
\hline & & \multicolumn{3}{|c|}{ PP + Negro de Fumo (1h) } & \multicolumn{3}{|c|}{$\begin{array}{c}\text { PP + Negro de Fumo + Pó de } \\
\text { Alumínio (1h) }\end{array}$} \\
\hline & Medidas & $\mathbf{1}\left({ }^{\circ} \mathbf{C}\right)$ & $2\left({ }^{\circ} \mathrm{C}\right)$ & $3\left({ }^{\circ} \mathbf{C}\right)$ & $\mathbf{1}\left({ }^{\circ} \mathbf{C}\right)$ & $2\left({ }^{\circ} \mathrm{C}\right)$ & $3\left({ }^{\circ} \mathbf{C}\right)$ \\
\hline Sensor 1 & & 70,0 & 69,3 & 68,1 & 66,8 & 69,4 & 69,9 \\
\hline Sensor 2 & & 58,5 & 57,5 & 56,6 & 56,0 & 58,1 & 58,7 \\
\hline Sensor 3 & & 53,4 & 54,9 & 53,6 & 53,0 & 55,5 & 56,5 \\
\hline Sensor 4 & & 36,0 & 35,6 & 34,8 & 33,3 & 35,6 & 36,6 \\
\hline \multirow[t]{3}{*}{ Sensor 5} & & 28,7 & 27,7 & 26,8 & 25,5 & 27,7 & 28,4 \\
\hline & & \multicolumn{3}{|c|}{ PP + Negro de Fumo (2h) } & \multicolumn{3}{|c|}{$\begin{array}{c}\text { PP + Negro de Fumo + Pó de } \\
\text { Alumínio }(2 \mathrm{~h})\end{array}$} \\
\hline & Medidas & $\mathbf{1}\left({ }^{\circ} \mathbf{C}\right)$ & $2\left({ }^{\circ} \mathrm{C}\right)$ & $3\left({ }^{\circ} \mathbf{C}\right)$ & $\mathbf{1}\left({ }^{\circ} \mathbf{C}\right)$ & $2\left({ }^{\circ} \mathrm{C}\right)$ & $3\left({ }^{\circ} \mathbf{C}\right)$ \\
\hline Sensor 1 & & 70,2 & 69,1 & 70,4 & 67,9 & 69,7 & 70,0 \\
\hline Sensor 2 & & 59,4 & 57,9 & 58,7 & 57,2 & 58,4 & 58,9 \\
\hline Sensor 3 & & 53,9 & 54,9 & 56,1 & 54,6 & 56,1 & 56,7 \\
\hline Sensor 4 & & 36,6 & 36,3 & 37,2 & 35,5 & 36,2 & 36,9 \\
\hline Sensor 5 & & 29,0 & 28,0 & 29,0 & 27,5 & 28,0 & 28,6 \\
\hline
\end{tabular}

Tabela 3 - Valores médios e desvio padrão das medidas realizadas.

\begin{tabular}{ccccc}
\hline & \multicolumn{2}{c}{ PP puro (1h) } & \multicolumn{2}{c}{ PP + pó de fumo (1h) } \\
\hline & Média $\left({ }^{\circ} \mathbf{C}\right)$ & Desvio padrão & Média $\left({ }^{\circ} \mathbf{C}\right)$ & Desvio padrão $\left({ }^{\circ} \mathbf{C}\right)$ \\
Sensor 1 & 69,133 & 0,404 & 68,067 & 1,724 \\
Sensor 2 & 57,867 & 0,503 & 56,400 & 2,095 \\
Sensor 3 & 51,700 & 1,735 & 53,267 & 1,930 \\
Sensor 4 & 35,800 & 1,058 & 34,500 & 2,081 \\
Sensor 5 & 29,000 & 0,656 & 26,133 & 1,762 \\
\hline & & PP puro $(\mathbf{2 h})$ & $\mathbf{P P}+$ pó de fumo $(\mathbf{2 h})$ \\
\hline Sensor 1 & Média $\left({ }^{\circ} \mathbf{C}\right)$ & Desvio padrão & Média $\left({ }^{\circ} \mathbf{C}\right)$ & Desvio padrão $\left({ }^{\circ} \mathbf{C}\right)$ \\
Sensor 2 & 69,567 & $\left({ }^{\circ} \mathbf{C}\right)$ & & 1,724 \\
Sensor 3 & 58,400 & 0,252 & 69,400 & 2,095 \\
Sensor 4 & 53,600 & 0,300 & 58,200 & 1,930 \\
Sensor 5 & 36,933 & 0,300 & 55,300 & 2,081 \\
\hline & 29,567 & 0,351 & 36,667 & 1,762 \\
\hline
\end{tabular}

PP + Negro de Fumo (1h) PP + Negro de Fumo + Pó de

Alumínio (1h)

Média $\left({ }^{\circ} \mathbf{C}\right) \quad$ Desvio padrão $\quad$ Média $\left({ }^{\circ} \mathbf{C}\right) \quad$ Desvio padrão $\left({ }^{\circ} \mathbf{C}\right)$

$\left({ }^{\circ} \mathrm{C}\right)$

$\begin{array}{lllll}\text { Sensor } 1 & 69,133 & 0,961 & 68,700 & 1,664\end{array}$




\begin{tabular}{ccccc} 
Sensor 2 & 57,533 & 0,950 & 57,600 & 1,418 \\
Sensor 3 & 53,967 & 0,814 & 55,000 & 1,803 \\
Sensor 4 & 35,467 & 0,611 & 35,167 & 1,692 \\
Sensor 5 & 27,733 & 0,950 & 27,200 & 1,513 \\
\hline & PP + Negro de Fumo (2h) & \multicolumn{2}{c}{ PP + Negro de Fumo + Pó de } \\
& & & \multicolumn{2}{c}{ Alumínio $(\mathbf{2 h})$} \\
\hline & Média $\left({ }^{\circ} \mathbf{C}\right)$ & Desvio padrão & Média $\left({ }^{\circ} \mathbf{C}\right)$ & Desvio padrão $\left({ }^{\circ} \mathbf{C}\right)$ \\
& & $\left({ }^{\circ} \mathbf{C}\right)$ & & 1,136 \\
Sensor 1 & 69,900 & 0,700 & 69,200 & 0,874 \\
Sensor 2 & 58,667 & 0,751 & 58,167 & 1,082 \\
Sensor 3 & 54,967 & 1,102 & 55,800 & 0,700 \\
Sensor 4 & 36,700 & 0,458 & 36,200 & 0,551 \\
\hline
\end{tabular}

\begin{tabular}{lcc}
\hline & \multicolumn{2}{c}{ Temperaturas experimento sem placa } \\
\hline Sensor 1 & $\mathbf{1 h}\left({ }^{\circ} \mathbf{C}\right)$ & $\mathbf{2 h}\left({ }^{\circ} \mathbf{C}\right)$ \\
Sensor 2 & 66,3 & 66,5 \\
Sensor 3 & 55,7 & 55,8 \\
Sensor 4 & 43,5 & 43,5 \\
Sensor 5 & 41,2 & 41,4 \\
& 35,6 & 36,0
\end{tabular}

Com os resultados obtidos foram determinados os percentuais de diminuição de temperatura entre os sensores 3 e 4 (localizados antes e depois da placa), os quais estão ligados à energia absorvida pela placa. Deve-se considerar ainda que entre as distâncias dos dois sensores em questão as medidas realizadas sem placa indicaram diminuição de $5 \%$ da temperatura causada por outros processos, além da absorção de energia das placas.

$\mathrm{Na}$ Tabela 4 são apresentados os percentuais de diminuição de temperatura entre os sensores 3 (209,3 mm de distância vertical da fonte) e 4 (249,6 mm de distância vertical da fonte).

Tabela 4 - Percentuais de diminuição de temperatura entre os sensores 3 e 4.

\begin{tabular}{cc} 
Placas & $\begin{array}{c}\text { Percentual de diminuição de } \\
\text { temperatura (\%) }\end{array}$ \\
\hline PP puro (1h) & 30,8 \\
PP puro (2h) & 31,1 \\
PP + pó de fumo (1h) & 35,2 \\
PP + pó de fumo (2h) & 33,7 \\
PP + Negro de Fumo (1h) & 34,3
\end{tabular}


PP + Negro de Fumo (2h)

PP + Negro de Fumo + Pó de Alumínio (1h)

PP + Negro de Fumo + Pó de Alumínio (2h)
33,2

36,1

35,1

Na Figura 5 é apresentado o gráfico das temperaturas médias em função da distância a partir da fonte para os 5 pontos de medida (sensores) após 1h para as 4 placas avaliadas e sem placa. E na Figura 6, após 2h do início do experimento.

Figura 5 - Resultados de temperatura nos 5 sensores após 1 h de experimento.

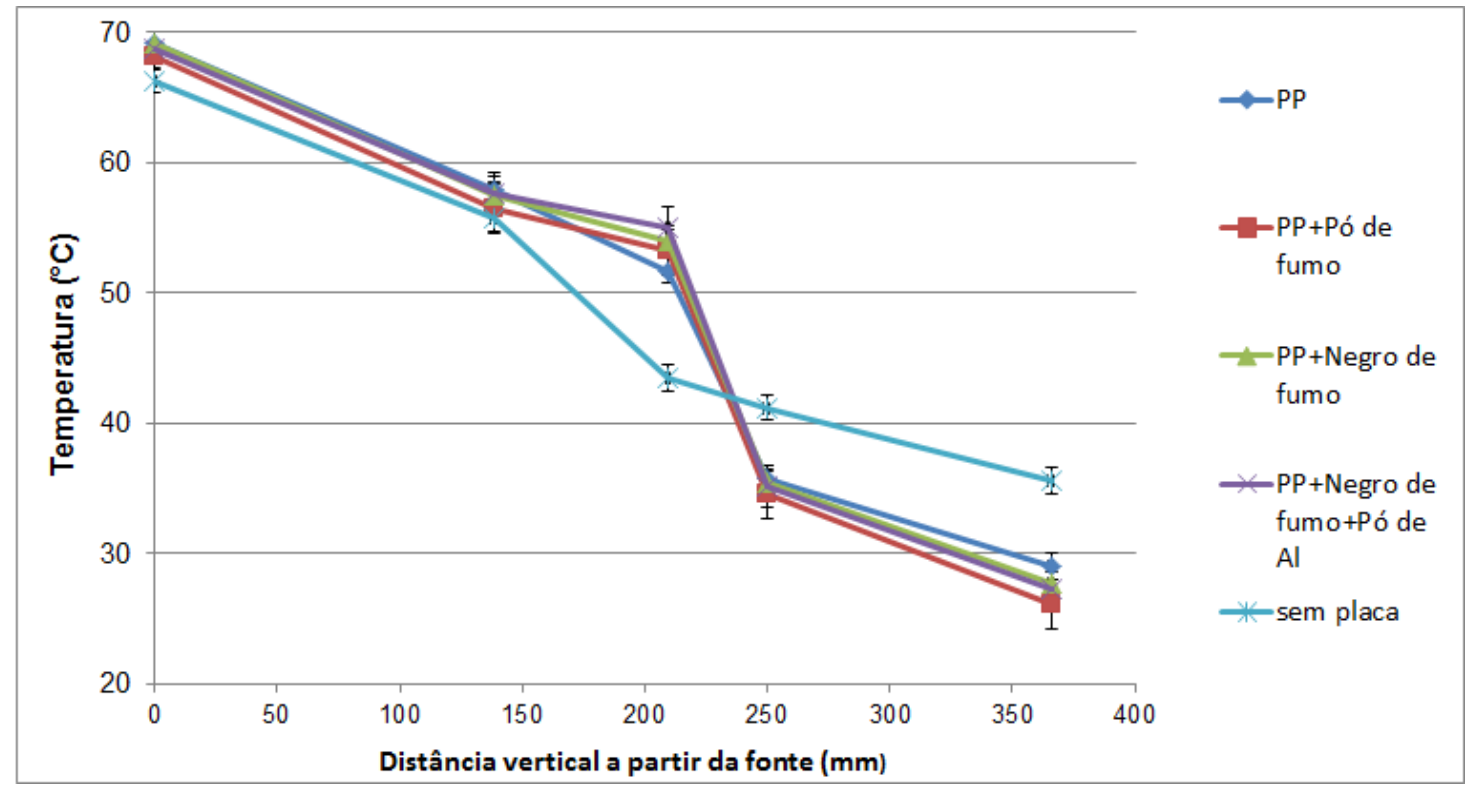

Figura 6 - Resultados de temperatura nos 5 sensores após 2 h de experimento.

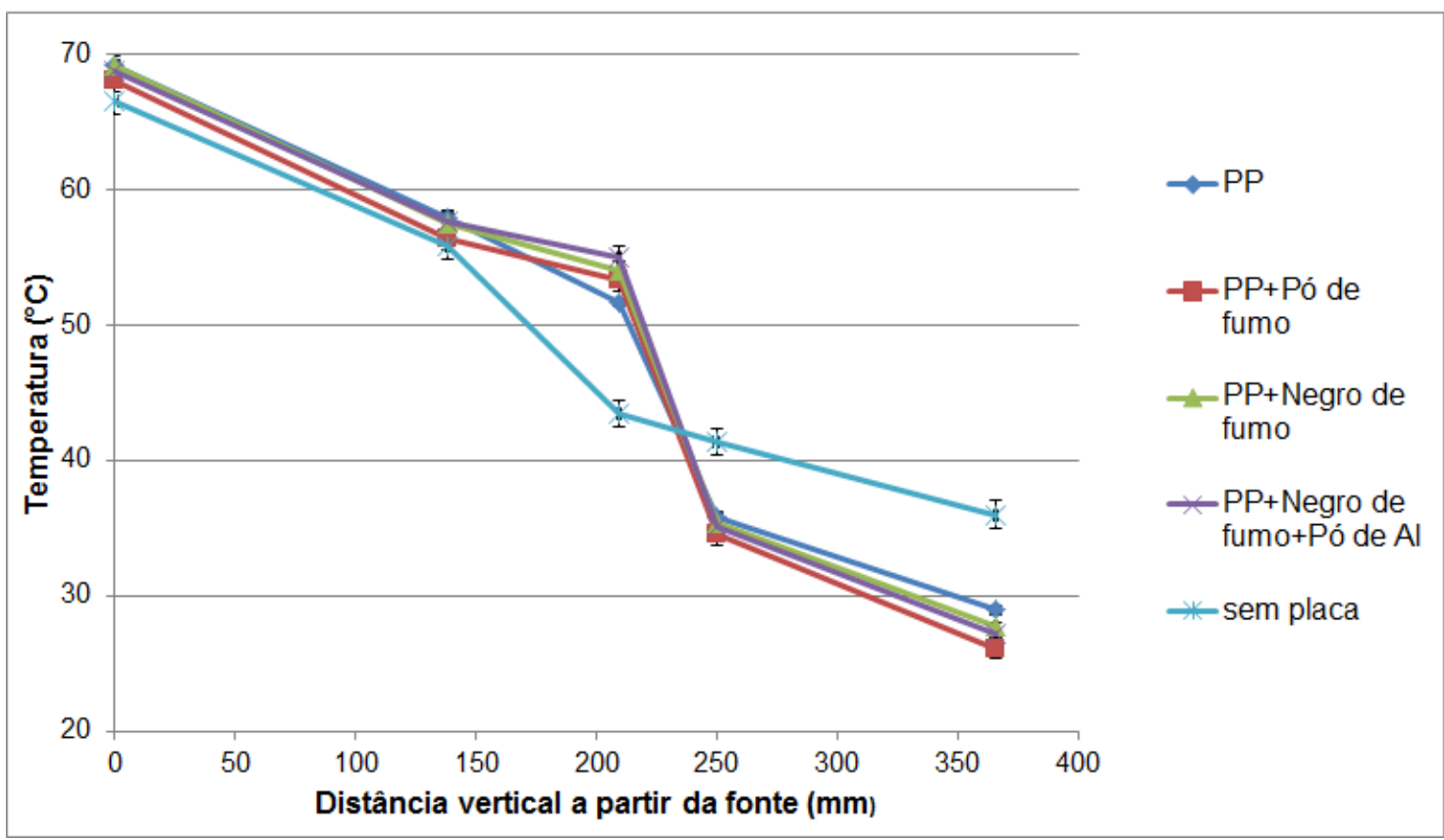


Para melhor visualização do comportamento das temperaturas entre os sensores 3 e 4 (antes e após a placa), nos gráficos das Figuras 7 e 8 são apresentadas as medidas apenas destes dois pontos para 1 e $2 \mathrm{~h}$ de experimento, respectivamente.

Figura 7 - Resultados de temperatura nos sensores 3 e 4 após 1 h de experimento.

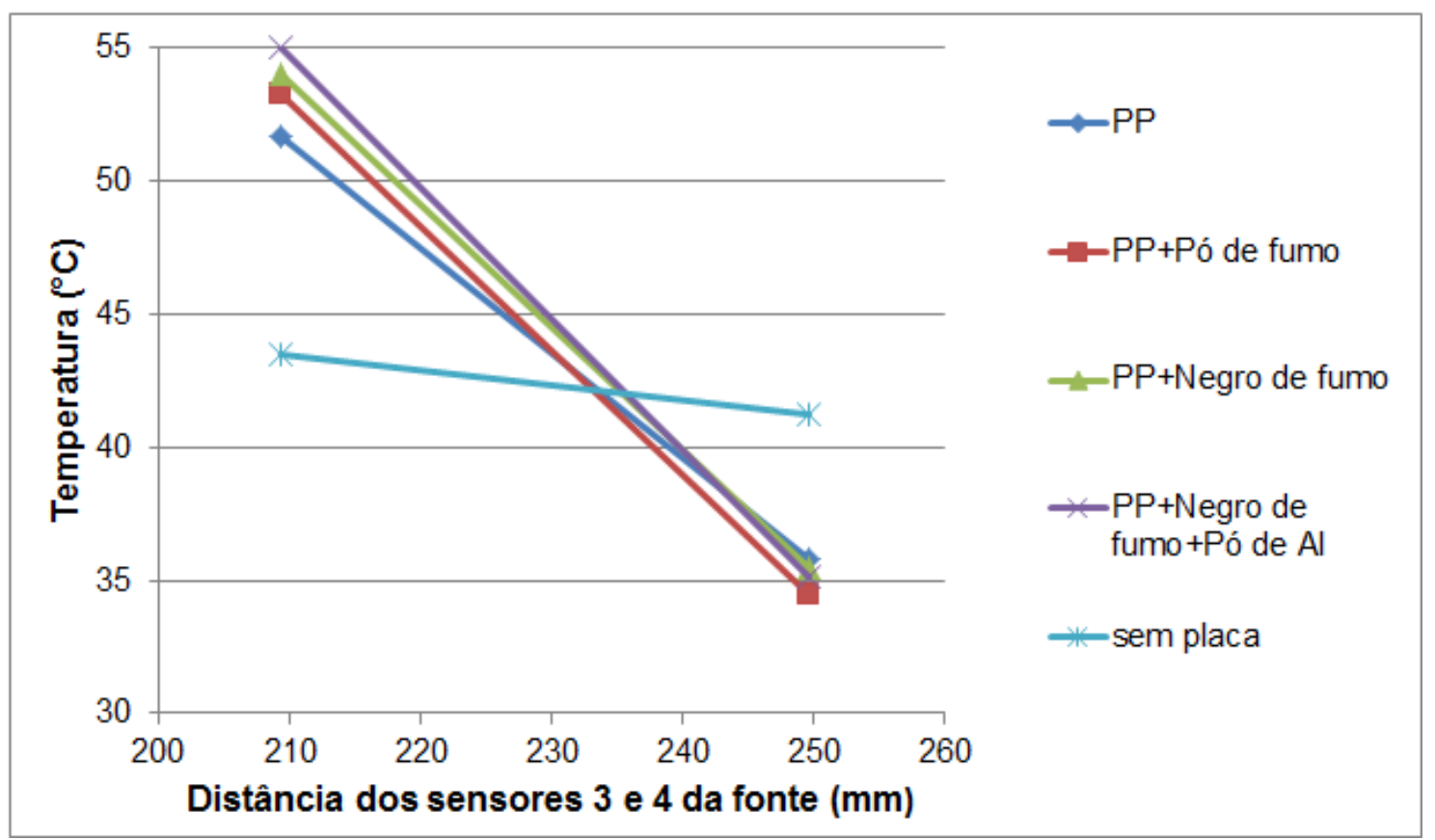

Figura 8 - Resultados de temperatura nos sensores 3 e 4 após 2 h de experimento.

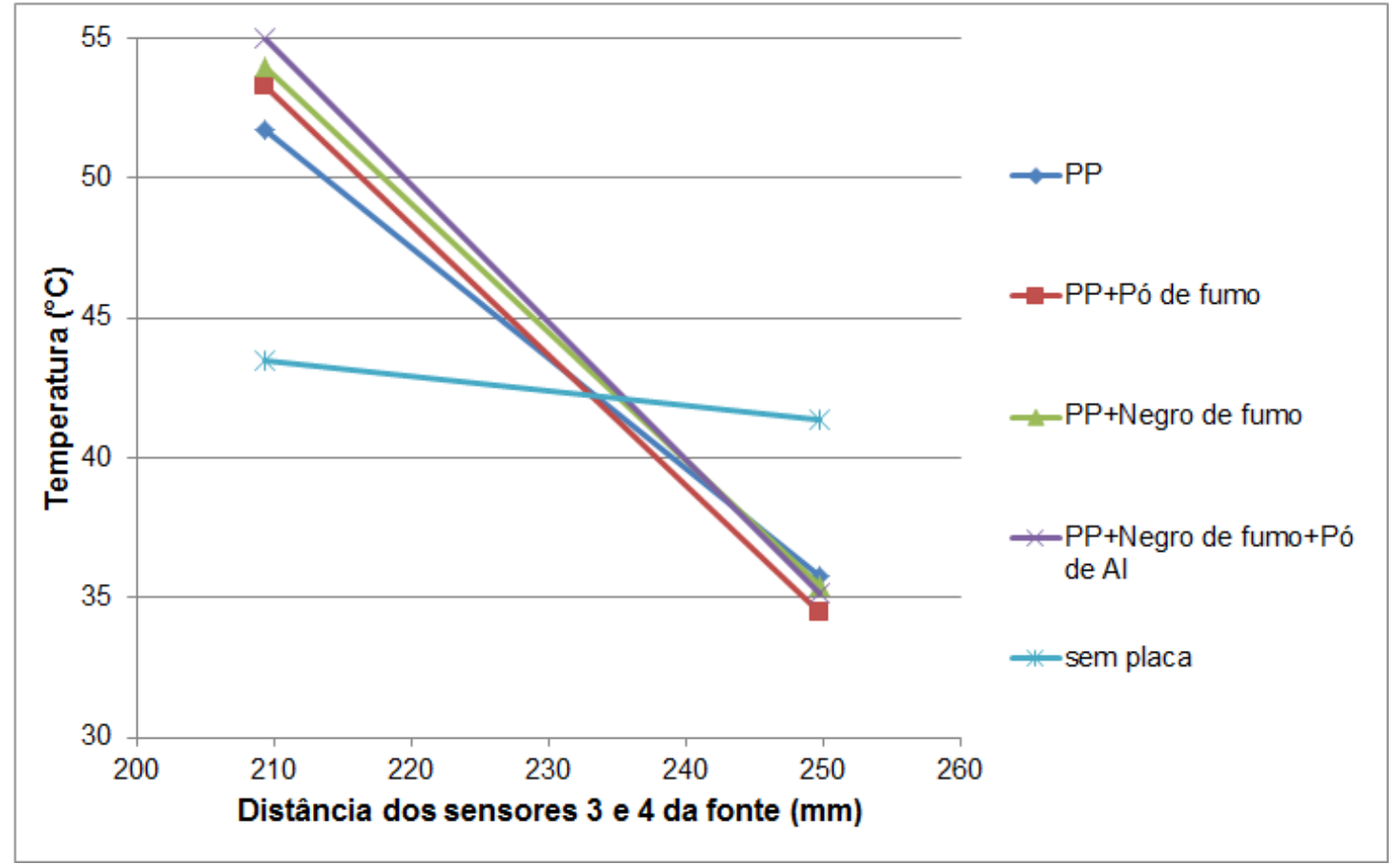

Os resultados apresentados nos gráficos anteriores (Figuras de 5 a 8) indicaram que com a colocação das placas o comportamento (medidas nos sensores 3 e 4) das temperaturas 
medidas é completamente modificado para qualquer uma das placas testadas. Isto é evidenciado nas Figuras 7 e 8, uma vez que as medidas sem placa apresentaram um comportamento quase linear para os sensores 3 e 4. Comparando as demais medidas, observaram-se variações vinculadas às distâncias, porém bem menores. Entre as placas avaliadas, verificou-se maior absorção (maior perda de temperatura) de calor pela placa de compósito de negro de fumo + pó de alumínio em sua composição, tanto em 1 hora como em 2 horas de teste realizado.

Aspectos positivos notados nos ensaios foram os dados obtidos pelas placas poliméricas com pó de fumo (tabaco). Visualizou-se nos gráficos uma boa absorção de calor também para esta formulação. Isto é interessante considerando que em sua composição há um rejeito das indústrias processadoras de tabaco e que é gerado em grandes quantidades, se tornando um problema para o descarte deste material.

Na Figura 9 estão representados graficamente os percentuais de diminuição de temperatura observada considerando os valores medidos nos sensores 3 e 4, que está vinculada à energia absorvida pela placa. A taxa de 5\% de diminuição é referente ao aparato experimental (perdas por irradiação e convecção), uma vez que foi observada sem placa na montagem experimental.

\section{Figura 9 - Percentual de diminuição de temperatura considerando a energia absorvida} pela placa.

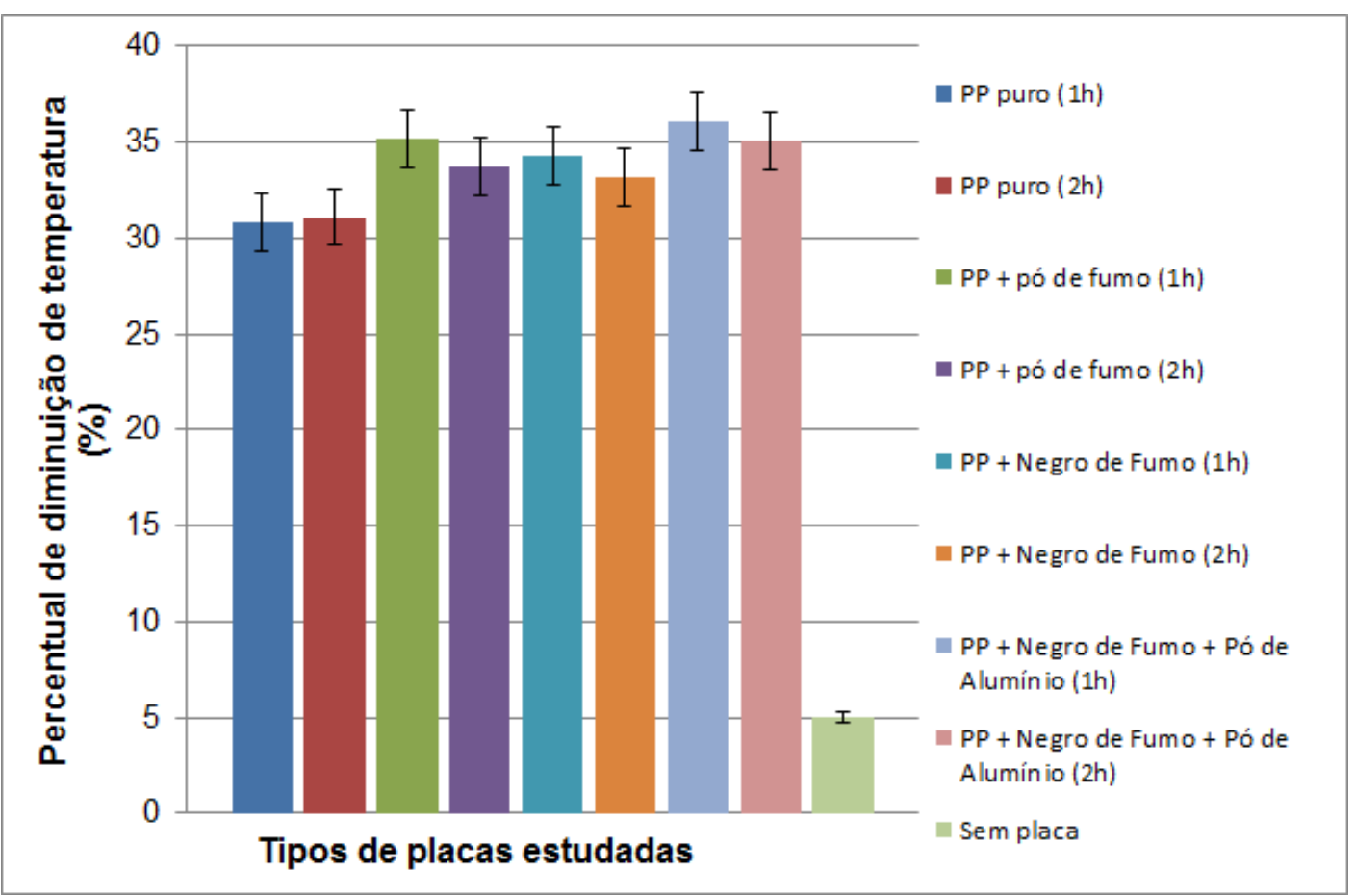


Os percentuais de diminuição de temperatura indicaram melhor desempenho para aplicação como placa absorvedora de radiação em aquecedores solares a que apresenta em sua composição negro de fumo e pó de alumínio. Ainda, salienta-se que a composição com pó de fumo apresentou melhores resultados que a com apenas negro de fumo. O pó de alumínio (melhor condutor de calor) foi o diferencial entre estas duas formulações. Até mesmo as placas com polipropileno puro apresentaram valores interessantes nas avaliações realizadas.

\section{CONSIDERAÇÕES FINAIS}

Todas as formulações estudadas nesta pesquisa apresentaram diminuição de temperatura maior que $30 \%$ daquela logo acima da placa, sendo que a formulação que envolveu $2 \%$ de pó de alumínio foi a que apresentou maior variação entre as temperaturas acima e abaixo da placa. Esta diminuição de temperatura está diretamente vinculada à quantidade de energia térmica absorvida por cada placa.

Foi observado que a utilização de pó de fumo (resíduo da indústria fumageira regional) na composição das placas foi interessante, uma vez que melhorou a absorção de energia comparada à placa de polipropileno puro. Dentro dos limites de temperaturas estudadas não foram observadas deformações nas placas testadas. Este é um ponto que deverá ser futuramente avaliado, uma vez que em coletores tradicionais para linha residencial de aquecimento de água chega-se no máximo a $100^{\circ} \mathrm{C}$.

Para a próxima etapa do projeto, serão realizados testes envolvendo novas formulações, tratamento e modelagem dos resultados, avaliação de placas com material tradicional, variação das especificações da fonte e maiores tempos de exposição das placas.

\section{AGRADECIMENTOS}

Agradecemos ao apoio recebido do Polo de Modernização e Inovação Tecnológica do Vale do Rio Pardo, e da Secretaria de Ciência, Inovação e Tecnologia do Estado do Rio Grande do Sul, e ao programa de iniciação científica da UNISC - Bolsa PUIC.

\section{REFERÊNCIAS}

ASTROSOL. Aquecedores. Disponível em: <http://www.astrosol.com.br/produtos/nossosprodutos>. Acesso em: 01 de dez. de 2013.

COMETTA, E. Energia solar: utilização e empregos práticos. São Paulo: Hemus, 127 p, 1978. 
MOAD, G. Synthesis of polyolefin graft copolymers by reactive extrusion. Progress in Polymer Science, v. 24, n. 1, p. 81-142, 1999.

PASSAGLIA, E.; COIAI, S.; CICOGNA, F.; CIARDELLI, F. Some recent advances in polyolefin functionalization. Polymer International, v. 63, n. 1, p. 12-21, 2014.

PORTAL DE ENERGIA RENOVÁVEIS. Energia Solar. Disponível em: <www.energiasrenovaveis.com>. Acesso em: 03 de dez. de 2013.

RUSSEL, L. D.; GUVAN, H.M. Modeling and analysis of a flat plate solar collector totally plastic. Journal Solar Enga. Energy, v. 104, p. 333-339, 1982.

SOLETROL. Aquecedores solares de água. Disponível em: 〈www.soletrol.com.br〉. Acesso em: 05 de dez. de 2013.

TSILINGIRIS, P.T. Design, analysis and performance of low-cost plastic film large solar water heating systems. Solar Energy, v. 60, n. 9, p. 245-256, 1997. 